\title{
Asymmetric amplification in amino acid sublimation involving racemic compound to conglomerate conversion $\dagger$
}

\author{
Cristóbal Viedma, ${ }^{* a}$ Wim L. Noorduin, $\ddagger^{a}$ José E. Ortiz, ${ }^{b}$ Trinidad de Torres ${ }^{b}$ and \\ Pedro Cintas ${ }^{c}$
}

\begin{abstract}
A straightforward unprecedented sublimation protocol that reveals both conversion of a racemic compound into a racemic conglomerate and subsequent enantioenrichment has been developed for the proteinogenic amino acid valine. The phenomenon has been observed in closed and open systems, providing insight into asymmetric amplification mechanisms under presumably prebiotic conditions.
\end{abstract}

Searching for new mechanisms of mirror-symmetry breaking at a molecular level is of primary importance to unveil the origin and evolution of single chirality on Earth. ${ }^{1}$ According to long held views, some kind of highly efficient and inexorable asymmetric amplification process would have operated to generate any visible enantiomeric excess (ee) thus paving the way to biomolecular homochirality. ${ }^{2}$

Much interest has emerged recently from abrasive grinding of a mixture of two enantiomorphous crystals that affords a complete transformation to a solid state of single chirality; one enantiomer completely disappears in an irreversible autocatalysis that nurtures the opposite one. ${ }^{3,4}$ Also enantioenrichment can be achieved by using physical equilibria (liquidsolid, gas solid, or even liquid-liquid). This involves selfdisproportionation or fractionation processes where enantioenriched mixtures lead ultimately to an unequal distribution of enantiomers between the different phases. ${ }^{5}$ Enantioenrichment in gas-solid systems represents a less travelled path than enantioselection in a solution-solid mixture. Results so far are promising, ${ }^{6-10}$ but the situation with some primeval amino acids is somewhat puzzling and interpretations largely depend on sample preparations. $\S$ Unfortunately, in these studies the collected sublimate that was investigated only represents a small fraction of the total amount of material. The underlying principle behind such fractionations lies in the distinctive nature of the intermolecular interactions between a racemic compound (D and L molecules crystallize in the same heterochiral crystal) and its enantiomorphic crystals. In general, the overwhelming

${ }^{a}$ Departamento de Cristalografía y Mineralogía, Facultad de Geología, Universidad Complutense, 28040 Madrid, Spain.

E-mail: viedma@geo.ucm.es; Fax: +34 91-394-4872;

Tel: + 34 91-394-4882

${ }^{b}$ Laboratorio de Estratigrafía Biomolecular, Escuela Técnica Superior de Ingenieros de Minas, 28040 Madrid, Spain

${ }^{c}$ Departamento de Química Orgánica e Inorgánica, Facultad de

Ciencias-UEX, E-06006 Badajoz, Spain

$\dagger$ Electronic supplementary information (ESI) available: Sublimation protocol and chiral HPLC analysis of valine samples. See DOI: $10.1039 / \mathrm{c} 0 \mathrm{cc} 04271 \mathrm{~d}$

$\ddagger$ Present address: School of Engineering and Applied Sciences, Harvard University, Cambridge, MA, USA. majority of chiral molecules crystallize as racemic compounds. However, to perform an enantiomeric resolution, the formation of racemic conglomerates is a prerequisite ( $D$ and $\mathrm{L}$ molecules crystallize in different crystals). Therefore a lot of effort has been undertaken to convert racemic compounds in racemic conglomerates. So far, there have been successful reports about these conversions in the solution, ${ }^{11,12}$ but the sublimation from the gas phase has hardly been explored to make racemic conglomerates.

Here we demonstrate that the amino acid valine, which crystallizes as a racemic compound, is first converted via sublimation into a racemic conglomerate that upon further heating enriches in the major enantiomer. Valine was chosen for this study as this proteinogenic amino acid was found to be non-racemic in meteoritic samples and the enantiomeric excesses closely match the values obtained by asymmetric photolysis under simulated interstellar conditions. ${ }^{13}$ Racemic compound crystals of valine were heated in tightly closed vessels (see ESI $\dagger$; the measured temperature at the bottom in contact with the conductive heater was $430{ }^{\circ} \mathrm{C}$ ). Almost instantly all solid material sublimes and condenses on the walls of the vessel, the coldest zone of the system. Then, this initially formed sublimation ring moved to the higher parts of the flask (Fig. 1).

Surprisingly, powder X-ray diffraction spectra (DebyeScherrer diagrams) demonstrated that the racemic compound crystals of valine are transformed into a racemic conglomerate phase during the sublimation-condensation process (Fig. 2). ${ }^{14,15}$

Bearing an asymmetric sublimation in mind, the process was repeated starting from an enantioenriched mixture with $40 \%$

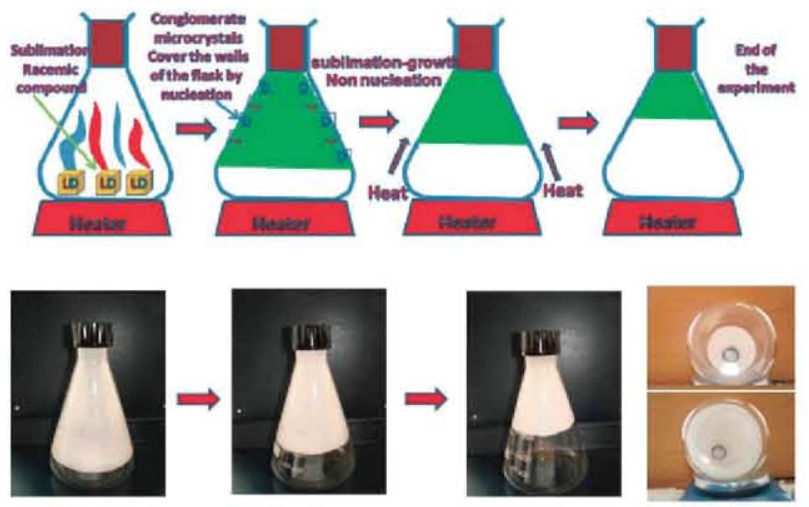

Fig. 1 top: Schematic drawing of the nucleation-condensation and sublimation-crystal growth observed during valine sublimation; bottom: temporal evolution of sublimation-growth in real systems (left), views from the bottom (right). 

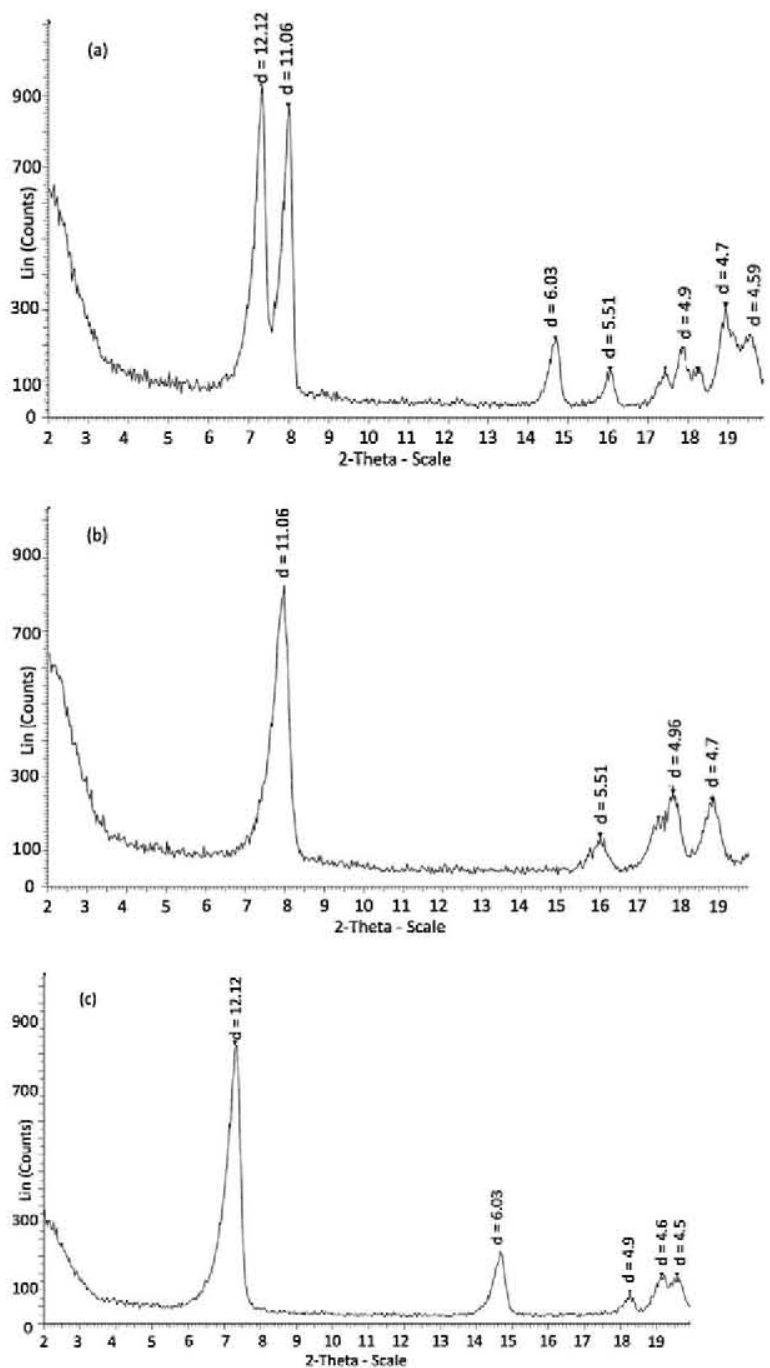

Fig. 2 Powder XRD diffractograms of (a) coexisting valine conglomerate (i.e. the $50: 50$ mechanical mixture of $\mathrm{D}$ - and L-valine) plus $\mathrm{D}, \mathrm{L}$-valine (i.e. racemic compound); (b) valine before sublimation (racemic compound); (c) solid obtained after sublimation (racemic conglomerate).

ee, generated from D,L-valine (racemic compound crystals) and L-valine. Chiral HPLC analysis did not reveal any enantioenrichment after the initial sublimation. Interestingly, however, when the subsequent sublimation and crystal growth occurred causing the movement of the sublimation ring to the higher parts of the vessel, the entire crystalline mass enriched up to $56 \%$ ee. As expected, starting with an excess in D- instead of L-valine resulted in an enrichment in D-valine. Furthermore, similar enrichment was observed starting from the conglomerate crystals of valine; that is when the enantiomerically enriched starting material was prepared from both enantiopure D- and L-valine crystals.

Close examination of the enantioenrichment at different positions of the sublimate revealed a gradual increase of the ee from $46 \%$ at the top of the flask up to $80 \%$ ee at the bottom of the sublimate (Fig. 3 (top), Table 1). The total amount of recovered sublimated material was almost $100 \%$, suggesting an overall enantioenrichment rather than segregation or selective enantiomer decomposition. To further investigate this

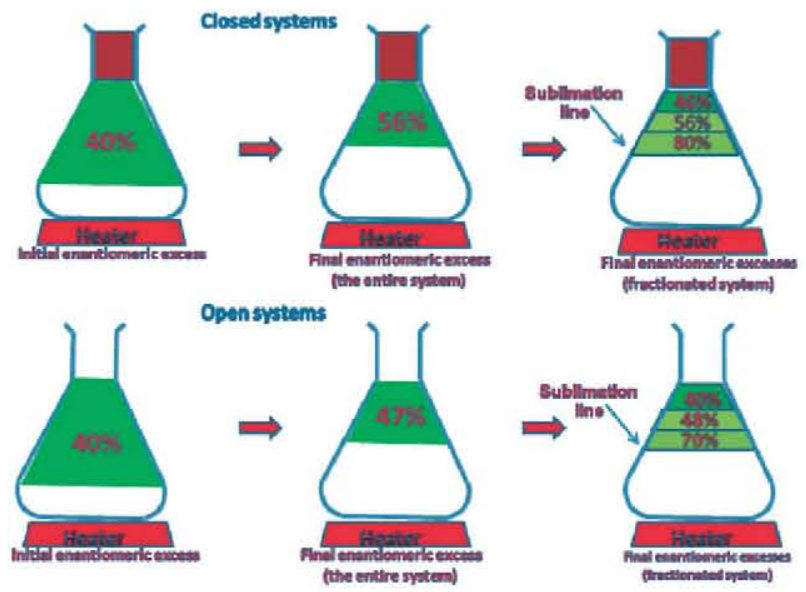

Fig. 3 Gradient-like evolution of ee's in valine sublimation from a conglomerate phase in (top) closed and (bottom) open flasks.

Table 1 Enantiomeric excesses after sublimation of valine (initial mixture with $40 \%$ ee) ${ }^{a}$

\begin{tabular}{lll}
\hline Sublimation stage & $\begin{array}{l}\text { ee closed } \\
\text { system }(\%)\end{array}$ & $\begin{array}{l}\text { ee open } \\
\text { system }(\%)\end{array}$ \\
\hline $\begin{array}{l}\text { Nucleation (whole system) } \\
\text { Sublimation + crystal growth }\end{array}$ & 40 & 40 \\
(whole system) & 56 & 47 \\
${\text { Zone } \mathrm{A}^{b}}_{\text {Zone }}{ }^{b}$ & 80 & 70 \\
Zone $^{b}$ & 56 & 48 \\
& 46 & 40
\end{tabular}

${ }^{a}$ Measured, after amino acid derivatization, on a Hypersil BDS C18 reverse-phase column (see ESI $\dagger$ for details). ${ }^{b}$ Longitudinal zones measured from the sublimation line.

intriguing behavior, the experiment was repeated in an open system, in which a eutectic gas phase would really be difficult to form. \| Similar amplification patterns were observed, although with slightly lower ee's (Fig. 3 (bottom), Table 1). It is worth noting that the whole sublimation-growth protocol took less than 12 min (see $\mathrm{ESI} \dagger$ ).

Overall, the sublimation of enantioenriched valine involves two distinct processes. First, during the sublimation of the valine, crystals undergo a recrystallization from a racemic compound into a racemic conglomerate. Secondly, on prolonged heating of the sublimated crystals, the sublimate rises up to the walls of the vessel and undergoes a substantial enrichment in the enantiomeric excess, particularly at the lower parts of the product.

It is known that all the hydrophobic amino acids crystallize in alternating hydrophilic/hydrophobic layers, a fact that usually prevents resolution by entrainment. ${ }^{16}$ One enantiomorph can thus operate as template for the crystallization of the other, yet obtaining the racemic conglomerate. Since the crystals of D,Lvaline are actually disordered, ${ }^{17}$ they have not undergone spontaneous resolution. One may argue that the racemic conglomerate is more stable at high temperatures than the racemic compound. Moreover, the difference in vapor pressures between racemic crystals and their enantiomers may be sufficiently different, resulting in a driving force for nucleation of the second crystal structure.

The second process that occurs during this particular sublimation is the gradual enantioenrichment from the 
sublimation line, which is observed both in the closed and open systems. Since no appreciable decomposition is observed during the process, the enrichment may be attributed to an asymmetric amplification mechanism that converts one enantiomer into the other one. Several amplification mechanisms can contribute to these observations. A process is required to drive the enantioenrichment of the major population and this process must depend on the concentration of crystals of the same handedness. Like in attrition-enhanced deracemizations, tiny crystal fragments of subcritical size, yet retaining their chiral sense, may be incorporated into a dominant population of crystals, thereby offering a greater surface area for further growth. ${ }^{18-21}$ On the other hand, also an enantioselective racemization may occur at the crystal surface, thus enhancing the growth of the major population in the solid phase..$^{22,23} * *$

In conclusion we have reported that the amino acid valine, racemic compound, is converted via sublimation into a conglomerate (note that no ee is needed for this). On continued heating the condensed crystals from sublimation undergo a substantial amplification of the initial ee, the phenomenon occurring in both closed and open systems. Although the enantiomeric imbalance obtained in a few minutes may seem modest as a whole, this process appears to be something interesting and different, and any sort of recycling of the enriched material could give total chiral purity. The case of valine may not be unique, $+\dagger$ but this substance itself provides a valuable template for enantioselective separations through gas-solid reactions.

The mechanistic chapter is forward-looking and poses several challenges, especially to elucidate the details of the observed enantioenrichment. Such studies are currently under way in our laboratories.

We acknowledge helpful discussions with active members of the European COST Action Systems Chemistry (CM0703). WLN thanks the EU COST Action CM0703 for a fellowship to conduct research at CV's laboratory. This work has been supported by a grant from the Spanish CICYT (Project CGL2009-10764).

\section{Notes and references}

$\S$ Serine for instance has a prominent and rather unique tendency for clustering under electrospray ionization. ${ }^{7}$ In the case of racemic serine crystals, no octamer $\left[\mathrm{Ser}_{8}+\mathrm{H}\right]^{+}$could be detected, while a mixture of D- and $\mathrm{L}$-serine gave a signal corresponding to that of pure $\mathbf{L}$-serine. The ee observed for leucine in the sublimate is not surprising as L-amino acid crystals were added to racemic D,L-crystals before sublimation. ${ }^{8}$ Due to different lattice structures, L-amino acids sublimed preferentially, whereas racemic crystals remained in the solid phase.

T Powder XRD shows distinct bands for enantiopure samples and for the corresponding racemates but only when the latter are racemic compounds (i.e. true racemates).

|| Sublimation of derivatives, other than amino acids in closed systems, exhibits however ees consistent with the formation of a gas-phase with a eutectic composition. ${ }^{10}$
** It should be emphasized that enantioenrichment is obtained regardless of the starting chiral composition; that is, from an initial racemic compound plus one enantiomer in excess but also from a mixture of L-crystals and D-crystals of valine, for which no racemic compound is present at all. A selective enantiodecomposition could not be detected.

$\dagger$ Although in a completely different scenario, polymerization of racemic amino acids (e.g. D,L-valine) in aqueous solution leads to peptides of homochiral sequences, a process of potential prebiotic interest. $^{24}$

1 A. Guijarro and M. Yus, The Origin of Chirality in the Molecules of Life, Royal Society of Chemistry, Cambridge, UK, 2009, ch. 5.

2 M. M. Green and B. A. Garetz, Tetrahedron Lett., 1984, 25, 2831-2834; M. Bolli, R. Micura and A. Eschenmoser, Chem. Biol., 1997, 4, 309-320; T. Satyanarayana and H. B. Kagan, Angew. Chem., Int. Ed., 2009, 48, 456-494.

3 C. Viedma, Phys. Rev. Lett., 2005, 94, 065504; C. Viedma, Astrobiology, 2007, 7, 312-317; W. L. Noorduin, T. Izumi, A. Millemaggi, M. Leeman, H. Meekes, W. J. P. Van Enckevort, R. M. Kellogg, B. Kaptein, E. Vlieg and D. G. Blackmond, J. Am. Chem. Soc., 2008, 130, 1158-1159; C. Viedma, J. E. Ortiz, T. de Torres, T. Izumi and D. G. Blackmond, J. Am. Chem. Soc., 2008 , 130, 15274-15275.

4 W. L. Noorduin, E. Vlieg, R. M. Kellogg and B. Kaptein, Angew. Chem., Int. Ed., 2009, 48,9600-9606.

5 D. G. Blackmond and M. Klussmann, Chem. Commun., 2007, 3990-3996.

6 V. Soloshonok and K. D. Klika, I. Fluorine Chem., 2010, 131, 451-548 (special issue); P. Cintas, Angew. Chem., Int. Ed., 2008, 47, 2918-2920.

7 R. H. Perry, C. Wu, M. Nefliu and R. G. Cooks, Chem. Commun., 2007, 1071-1073.

8 S. P. Fletcher, R. B. C. Jagt and B. L. Feringa, Chem. Commun., $2007,2578-2580$.

9 A. Bellec and J.-C. Guillemin, Chem. Commun., 2010, 46, $1482-1484$.

10 A. Bellec and J.-C. Guillemin, J. Fluorine Chem., 2010, 131, 545-548.

11 C. Viedma, Origins Life Evol. Biosphere, 2001, 31, 501-509.

12 T. Lee and Y. K. Lin, Cryst. Growth Des., 2010, 10, 1652-1660.

13 U. Meierhenrich, Amino Acids and the Asymmetry of Life, Springer, Berlin, 2008, pp. 146-147.

14 E. L. Eliel and S. H. Wilen, Stereochemistry of Organic Compounds, Wiley, New York, 1994, p. 187.

15 C. P. Brock, W. B. Schweizer and J. D. Dunitz, J. Am. Chem. Soc., 1991, 113, 9811-9820.

16 M. Berfeld, D. Zbaida, L. Leiserowitz and M. Lahav, Adv. Mater., 1999, 11, 328-331.

17 S. Grayer Wolf, Z. Berkovitch-Yeilin, M. Lahav and L. Leiserowitz, Mol. Cryst. Liq. Cryst., 1990, 186, 3-17.

18 J. Crusats, S. Veintemillas and J. Ribó, Chem.-Eur. J., 2006, 12, $7776-7781$

19 M. Uwaha, J. Phys. Soc. Jpn., 2004, 73, 2601-2603.

20 J. M. McBride and J. C. Tully, Nature, 2008, 452, 161-162.

21 W. L. Noorduin, W. J. P. van Enckevort, H. Meekes, B. Kaptein, R. M. Kellogg, J. C. Tully, J. M. McBride and E. Vlieg, Angew. Chem., Int. Ed., 2010, 49,8435-8438.

22 Y. Saito and H. Hyuga, J. Phys. Soc. Jpn., 2008, 77, 113001.

23 S. Wei, M. Mauksch and S. B. Tsogoeva, Chem.-Eur. J., 2009, 15, $10255-10262$.

24 I. Rubinstein, G. Clodic, G. Bolbach, I. Weissbuch and M. Lahav, Chem.-Eur. J., 2008, 14, 10999-11009; I. Weissbuch, L. Leiserowitz and M. Lahav, Prebiotic Chemistry, Topics in Stereochemistry Vol. 259, ed. P. Walde, Springer, Berlin, 2005, pp. 123-165. 